\title{
Assessing the capacity for mental manipulation in patients with statically-determined mild cognitive impairment using digital technology
}

Sheina Emrani ${ }^{1}$, Melissa Lamar ${ }^{2}$, Catherine C. Price ${ }^{3}$, Satya Baliga ${ }^{4}$, Victor Wasserman ${ }^{1}$, Emily Matusz $^{5}$, Rod Swenson $^{6}$, Ganesh Baliga ${ }^{*}$, David J. Libon ${ }^{1,5 *}$

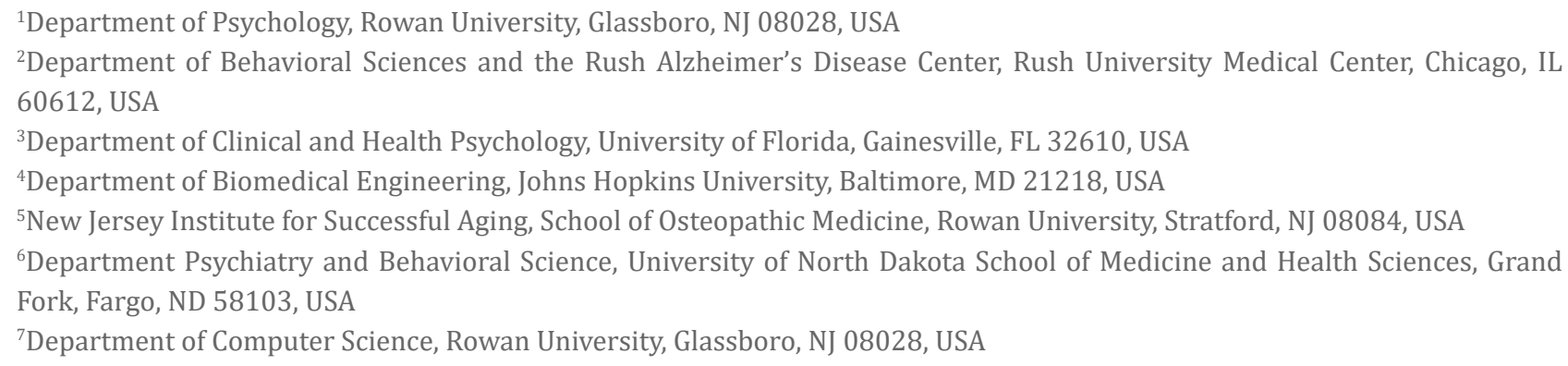

*Correspondence: Ganesh Baliga, Department of Computer Science, Rowan University, Glassboro, NJ 08028, USA. baliga@ rowan.edu; David J. Libon, New Jersey Institute for Successful Aging, School of Osteopathic Medicine, Rowan University, Stratford, NJ, 08084, USA. libon@rowan.edu

Academic Editor: Lindsay A. Farrer, Boston University School of Medicine, USA

Received: August 17, 2020 Accepted: February 2, 2021 Published: February 28, 2021

Cite this article: Emrani S, Lamar M, Price CC, Baliga S, Wasserman V, Matusz E, et al. Assessing the capacity for mental manipulation in patients with statically-determined mild cognitive impairment using digital technology. Explor Med. 2021;2:8697. https://doi.org/10.37349/emed.2021.00034

\begin{abstract}
Aims: Prior research employing a standard backward digit span test has been successful in operationally defining neurocognitive constructs associated with the Fuster's model of executive attention. The current research sought to test if similar behavior could be obtained using a cross-modal mental manipulation test.

Methods: Memory clinic patients were studied. Using Jak-Bondi criteria, 24 patients were classified with mild cognitive impairment (MCI), and 33 memory clinic patients did not meet criteria for MCI (i.e. non-MCI). All patients were assessed with the digital version of the WRAML-2 Symbolic Working Memory Test-Part 1 , a cross-modal mental manipulation task where patients hear digits, but respond by touching digits from lowest to highest on an answer key. Only 4 and 5-span trials were analyzed. Using an iPad, all test stimuli were played; and, all responses were obtained with a touch key. Only correct trials were analyzed. Average time to complete trials and latency for each digit was recorded.

Results: Groups did not differ when average time to complete 4-span trials was calculated. MCI patients displayed slower latency, or required more time to re-order the $1^{\text {st }}$ and $3^{\text {rd }}$ digits. Regression analyses, primarily involving initial and latter response latencies, were associated with better, but different underlying neuropsychological abilities. Almost no 5-span analyses were significant.
\end{abstract}


Conclusions: This cross-modal test paradigm found no difference for total average time. MCI patients generated slower $1^{\text {st }}$ and $3^{\text {rd }}$ response latency, suggesting differences in time allocation to achieve correct serial order recall. Moreover, different neuropsychological abilities were associated with different time-based test components. These data extend prior findings using a standard backward digit span test. Differences in time epochs are consistent with constructs underlying the model of executive attention and help explain mental manipulation deficits in MCI. These latency measures could constitute neurocognitive biomarkers that track emergent disease.

\section{Keywords}

Biomarkers, mild cognitive impairment, executive control, temporal organization, intra-component latency, digit span

\section{Introduction}

Dysexecutive impairment is common in patients with dementia syndromes such as Alzheimer's disease (AD) and vascular dementia (VaD), and in patients with mild cognitive impairment (MCI) [1-3]. Past research has shown that dysexecutive impairment in $\mathrm{VaD}$ associated with magnetic resonance imaging (MRI) white matter alterations and among some MCI patients is "context independent". This means that elements of a dysexecutive syndrome can be found across virtually all neurocognitive domains that might be assessed. Thus, on tests examining the production of graphomotor perseveration, patients with VaD tend to produce errors suggesting an inability to desist from a motor act. On tests that assess verbal concept formation (e.g., the Similarities subtest) patients with VaD and some MCI patients demonstrate a gross inability to assume an abstract attitude and treat each element of the word pair as a separate test stimulus (e.g., dog-lion: "one is a pet and the other roars"). When memory is assessed with the serial list-learning test, patients with executive impairment often present with retrieval, rather than consolidation problems.

By contrast, the dysexecutive syndrome associated with $\mathrm{AD}$ and other MCI patients is "context dependent", i.e. subordinate to other more pervasive neuropsychological problems. Among these patients, graphomotor perseverations have been shown to be highly associated with naming and lexical access problems. The errors made on tests assessing verbal concept formation tend to be vague, but retain some context to provide a supraordinate concept that binds the word pair (dog-lion: "I like them"). Serial-list learning test performance is often replete with elements consistent with amnesia (see ref 1-3 for a complete review). Focal deficits involving language and visuospatial operations can occur; however, these syndromes are comparatively rare $[4,5]$.

The research reviewed above provides useful heuristics to understand the constructs that underlie executive difficulty in dementia and MCI. Another heuristic mechanism that may be used to evaluate constructs that underlie executive difficulty in dementia and $\mathrm{MCI}$ is to assess performance as a function test epoch [6]. Patients with VaD and some MCI patients tend to produce a negative slope on executive tests when outcome is measured as a function of time $[3,6]$. Past research demonstrating this time-related, dysexecutive impairment has been interpreted within the context of Fuster's model of executive attention $[7,8]$. This model views the time necessary to bring a task to fruition as a temporal gestalt. Subordinate to this temporal gestalt are three separate, but highly integrated neurocognitive constructs that govern how time is allocated - working memory, preparatory set, and inhibitory control.

Working memory is memory for the short term and operates to marshal the necessary neurocognitive resources to initiate a response as requested. Preparatory set is involved in recruiting neurocognitive resources to sustain the necessary mental set to successfully complete the task at hand. Finally, inhibitory control denotes the capacity to filter extraneous stimuli such as perseverations and behavior resulting in the loss of mental set and to minimize the emergence of errors. Evidence supporting the existence of these three neurocognitive constructs has come from both imaging and primate research $[7,8]$. 
Recently, Emrani and colleagues [9, 10] administered an iPad version of the Backward Digit Span Test, originally described by Lamar and colleagues $[11,12]$, to patients with MCI versus patients with some, but insufficient cognitive deficits to meet diagnostic criteria for MCI (i.e. non-MCI). In this research, correct 5 -span test trials were analyzed. Latency, or reaction time, to repeat each individual digit backward was measured. The analysis of latency data found that when $\mathrm{MCI}$ and non-MCI patients scored 100 percent correct on these trials, there was no difference in the average total time to complete correct trials. However, there were distinct differences in the allocation of time $[9,10]$. Specifically, non-MCI patients tended to require more time, i.e. produced a slower latency, on the second and fourth responses on digits backwards. Comparatively, the MCI patients displayed slower latencies on position three as compared to non-MCI patients. Slower initial latencies were interpreted as a means to define operationally the neurocognitive construct of working memory. Thus, the slower latency on the second response suggests patients not meeting criteria for $\mathrm{MCI}$ were, perhaps, allocating greater neurocognitive resources to initially re-order test stimuli as required. By contrast, the latency to respond the third number backward was slower for MCI as compared to non-MCI patients. Emrani and colleagues $[9,10]$ interpreted this behavior as means to define operationally the neurocognitive construct of preparatory set, or the ability to sustain the necessary mental set to generate a correct response. Slower third response latency generated by MCI patients suggest that these patients essentially needed to work harder, i.e. marshal greater resources than their non-MCI counterparts to sustain the necessary mental set for correct responding. Finally, non-MCI patients produced a slower latency for the fourth digit repeated backward. Emrani and colleagues [9] interpreted this behavior to define operationally the neurocognitive construct of inhibitory control. That is to say, slower latency on position four by the non-MCI group may reflect a greater capacity to monitor behavior to prevent the production of errors. Most importantly, these data were interpreted to support the notion that it is possible to extract specific time epochs when asked to repeat numbers backward that can operationally define separate, but highly integrated neurocognitive constructs that underlie executive abilities in MCI.

In the current research, memory clinic patients were studied and assigned to two groups-patients with MCI using Jak-Bondi criteria [13]; and patients who presented with self-reported cognitive complaints but did meet criteria for MCI. These patients were labeled non-MCI. All patients were assessed with an iPad version of Symbolic Working Memory subtest from the Wide Range Assessment of Memory and Learning $2^{\text {nd }}$ Edition (WRAML-2) [14]. The overall goal of the current research was to extend the findings obtained with a standard backward digit span test, using a cross-modal test paradigm, i.e. a test that requires the management of a wider array of neurocognitive abilities. Thus, in the current research our intent was to assess how well a cross-modal test paradigm can operationally define the neurocognitive constructs that underlie the model of executive attention [7, 8] and differentiate patients with $\mathrm{MCI}$ from non-MCI when serial order responding is 100 percent correct.

\section{Methods}

\section{Participants}

All patients studied in the current research ( $n=57 ; 68.40 \%$ female) were clinical referrals to the New Jersey Institute for Successful Aging Memory Assessment Program (MAP). All MAP patients were administered a comprehensive neuropsychological evaluation and were evaluated by a social worker and a board-certified geriatric psychiatrist. A computed tomography (CT) or MRI study of the brain and appropriate blood serum tests were obtained to assess for reversible causes of dementia. Based on clinical interview, the score obtained from the Geriatric Depression Scale [15], and data from the Neuropsychiatric Inventory [16], no psychiatric co-morbidities were present. No patients in the current research presented with dementia.

A 2-step procedure was used to first diagnose then classify patients. First, all available information was reviewed at an interdisciplinary team conference to determine the presence of dementia, MCI, or some other clinical syndrome. Second, when patients were believed to be presenting with MCI, Jak-Bondi criteria [13] were applied as described below. Patients diagnosed with MCI presented with evidence of neuropsychological 
impairment relative to age and education, preservation of general functional abilities, and the absence of dementia. Some memory clinic patients who were clinically diagnosed with MCI did not present with the required level of cognitive impairment to meet Jak-Bondi criteria for MCI. These patients were labeled nonMCI. All patients described subjective complaints of cognitive difficulty. The decision to use Jak-Bondi [13] criterion is based on prior research showing that this diagnostic criterion limits diagnostic errors [17].

Information from 106 patients were reviewed. Patients were excluded because of diagnosis of a dementia syndrome $(n=35)$, depression $(n=6)$, suspected alcohol abuse $(n=4)$, and unstable medical problems such as a seizure disorder or suspected brain injury $(n=4)$. A knowledgeable family member was available to provide information regarding functional status for all patients. Patients were not compensated. Data were collected based on a Rowan University institutional review board (IRB) approved, retrospective chart review. This study was conducted consistent with the Declaration of Helsinki.

\section{Neuropsychological assessment}

The neuropsychological protocol used to classify MCI subtypes was the same as described by Emrani and colleagues [18]. Nine parameters drawn from three domains of cognition (executive control, naming/lexical access, and episodic memory) were expressed as z-scores derived from normative data (see Table 1 for full details). While we acknowledge that other neuropsychological tests/domains of cognitive functioning could have been used, the rationale for using the current protocol was based on extensive prior research showing that these tests are able to illustrate key neurocognitive constructs [19-21].

Table 1. Neuropsychological domains

\begin{tabular}{lll}
\hline Executive function domain & Language/lexical access domain & Episodic memory domain \\
\hline $\begin{array}{l}\text { WMS-Mental Control Subtest } \\
\text { (Boston revision) [6] }\end{array}$ & 60-item Boston NamingTest [36] & CVLT (short form)_Immediate Free Recall [38] \\
Letter Fluency_'FAS' [36] & Animal Fluency [36] & CVLT (short form)_Delayed Free Recall [38] \\
Trail Making Test-Part B [36] & WAIS-III Similarities Subtest [37] & CVLT (short form)_Delayed Recognition [38] \\
\hline
\end{tabular}

WMS: Wechsler Memory Scale; CVLT: California Verbal Learning Test; WAIS-III: Wechsler Adult Intelligence Scale-3 ${ }^{\text {rd }}$ edition

\section{Classifying MCI versus non-MCI}

MCI

Jak-Bondi criteria [13] were used to determine the presence of MCI $(n=24)$. Using these neuropsychologically derived criteria, single domain MCI was diagnosed when participants score $>1.0$ standard deviation below normative expectations on two of three measures within any single cognitive domain. Mixed MCI was diagnosed when participants scored $>1.0$ standard deviation below normative expectations on any two or more of the three measures across multiple cognitive domains. Because of the modest sample, MCI subtypes were not examined.

Non-MCI

Despite self-reported cognitive complaints, some patients did not meet Jak-Bondi criteria [13] for MCI as described above. These patients either scored above -1standard deviation (SD) on all nine neuropsychological parameters $(n=19)$; scored 1SD below the mean on only one measure $(n=7)$; or scored 1SD below on one measure within one cognitive, and 1 measure within another cognitive domain $(n=7)$. This group was label non-MCI $(n=33)$.

\section{iPad administration of the symbolic working memory test}

The Symbolic Working Memory subtest is part of the Wide Range Assessment of WRAML-2 [14]. The paper and pencil version of this this consists of two parts. On part 1 , numbers are administered and on part 2, a combination of numbers and letters are administered. The current research describes data obtained only from the Symbolic Working Memory-Part 1 subtest (i.e. numbers only). This Symbolic Working Memory subtest is comprised of three trials of 2, 3, 4, 5, and 6 digit span lengths. Each trial block contains 3 test trials, except for the 6-span block where only 2 trials are administered (total trials $=14$ ). For the standard paper and pencil version of this test, the administrator reads the numbers for each trial. Using a response 
key displaying the numbers 1 through 9, patients are asked to re-order the numbers administered by pointing to numbers from lowest to highest.

The iPad version of this test was administered using the same procedures as the paper and pencil test. The examiner initiated each test trial by pressing a start key on the iPad. Upon pressing the start key, the iPad played all numbers one second at a time. After test stimuli were administered, a response key immediately appeared. The response key was programmed to look exactly as the paper and pencil version. Patients were asked to touch the numbers from lowest to highest. Latency, or reaction time, elapsed starting immediately upon the appearance of the response key.

As stated above each trial block contained three trials. Patients tended to perform at ceiling on 2- and 3-span trial blocks (non-MCI correct 2-span trials $=2.87 \pm 0.33$; MCI correct 2-span trials $=3.00 \pm 0.00$; $F[1,56]=3.14 ; P=0.079$; non-MCI correct 3-span trials $=2.91 \pm 0.29$; MCI correct 3-span trials $=2.71 \pm$ $0.62 ; F[1,56]=2.63, P=0.110$ ). Also, patients tended to perform at floor on the 6-span trial block (non$\mathrm{MCI}$ correct trials $=0.64 \pm 0.74 ; \mathrm{MCI}$ correct trials $=0.33 \pm 0.48 ; F[1,56] ; P=0.086$ ). For these reasons, and because the 2- and 3- trial blocks do not provide sufficient time-based data, we analyzed only the 4- and 5 -span trial blocks.

\section{Intra-component latency and average total time for correct responses}

The model of executive attention [7, 8] suggests that successful mental manipulation is based, in part, in managing the time necessary to bring a task to fruition; and, the simultaneous operation(s) of all three neurocognitive constructs-working memory, preparatory set, and inhibitory control must operate within the context of temporal organization. Thus, to optimize our ability to assess all three constructs we chose to analyze only correct test trials, i.e. trials where serial order recall was 100 percent correct.

The iPad calculated average total time for each trial block by summing the total time for all correct trials from a trial block then dividing by the number of correct trials. Intra-component latency was measured and defined as the time elapsed to touch each digit as requested, i.e. time zero (when the response key appeared) to the first response, time from the end of the first response to the beginning of the second response etc.

\section{Statistical Analyses}

All continuous variables were screened for outliers and evaluated for departures of normality through quantitative examination of skewness and kurtosis, as well as visual inspection of frequency distributions. To address non-normality issues, where indicated, we assigned outliers a lower weight [22]. Post-hoc comparisons were made using $t$-tests. The Welch correction was used because of uneven sample size between groups. To guard against multiple comparisons significance was set at $P<0.01$.

Between- and within-group latency analyses

All analyses were carried out using SPSS. Between-group differences were assessed for the number of correct trials and average total time for correct trials. The effect of group on individual intra-component latencies was analyzed with two repeated measured ANOVAs, i.e. 2- group (non-MCI, MCI) by 4- or 5-span intra-component latencies.

Symbolic Working Memory latencies and Neuropsychological Test performance

Regression analyses were conducted to assess the association between neuropsychological test performance and Symbolic Working Memory intra-components latencies. For these analyses, executive functioning, naming/lexical access, and episodic memory index scores were calculated using the same neuropsychological parameters described above. Neuropsychological domain indices were calculated by averaging performance of all three tests within each domain. A total of six regression analyses were conducted, where each neuropsychological index score was the dependent variable and all 4- or 5-span intra-component latencies where the independent variables. 


\section{Results}

\section{Demographic Characteristics}

Demographic and clinical information can be found in Table 2. No between-group differences were found for age, education, the Geriatric Depression Scale [15] and projected premorbid general intellectual abilities assessed with the Wide Range Achievement Test Reading subtest-IV [23]. MCI patients scored marginally lower on the Mini-Mental State Examination $(F[1,39.45]=8.43, P<0.006, \eta 2=0.145$; [24]).

Table 2. Demographic and clinical information

\begin{tabular}{llll}
\hline & non- $\mathbf{M C l}(\boldsymbol{n}=\mathbf{3 3})$ & $\mathbf{M C l}(\boldsymbol{n}=\mathbf{2 4})$ & Significance \\
\hline Age & $73.90(7.93)$ & $72.37(5.37)$ & $\mathrm{ns}$ \\
Education & $15.63(2.21)$ & $15.04(2.64$ & $\mathrm{ns}$ \\
MMSE & $28.72(1.35)$ & $27.41(1.88)$ & $P<0.003$ \\
GDS & $2.36(2.47)$ & $2.75(2.11)$ & $\mathrm{ns}$ \\
WRAT-IV reading subtest & $115.09(16.29)$ & $110.04(16.47)$ & $\mathrm{ns}$ \\
\hline
\end{tabular}

MMSE: Mini-Mental State Examination; GDS: Geriatric Depression Scale; WRAT-IV: Wide Range Achievement Test; ns: not significant

\section{4- and 5-span trials, average total time, and intra-component latency}

Correct trials and average total time to complete trials

No differences were found for total correct 4-span trials. However, average total time to completion for the 4-span trial block was greater for MCI compared to non-MCI groups $(F[1,32.80]=9.85 ; P<0.004 ; \eta 2=$ 0.176). For the 5-span trial block, the opposite pattern emerged. Specifically, groups differed on total correct trials such that non-MCI patients produced more correct 5 -span trials as compared to the MCI group (F[1, $35.23]=7.27, P<0.011 ; \eta 2=0.133$ ). However, there was no between-group difference for the average total time on correct 5 -span trials (Table 3 ).

Table 3. Symbolic working memory—correct trials and average total time to completion

\begin{tabular}{llll}
\hline & non- $\mathbf{M C I}(\boldsymbol{n}=\mathbf{3 3})$ & $\mathrm{MCl}(\boldsymbol{n}=\mathbf{2 4})$ & significance \\
\hline 4-span correct trials (range 0-3) & $2.82(0.39)$ & $2.58(0.65)$ & $\mathrm{ns}$ \\
4-span average total time (secs.) & $5.80(2.03)$ & $8.44(3.74$ & $P<0.001$ \\
5-span correct trials (range 0-3) & $2.21(0.74)$ & $1.46(1.21)$ & $P<0.005$ \\
5-span average total time (secs.) & $8.72(3.71)$ & $10.81(4.91)$ & $\mathrm{ns}$ \\
6-span correct trials (range 0-3) & $0.64(0.74$ & $0.33(0.48)$ & $\mathrm{ns}$ \\
6-span average total time (secs.) & $11.41(5.25)$ & $11.62(4.63)$ & $\mathrm{ns}$ \\
\hline
\end{tabular}

ns: not significant

\section{Group by 4-span intra-component latency}

The group by serial order intra-component latencies repeated measures ANOVA yielded a marginally significant group by serial order position interaction $(F[3,53]=3.24, P<0.029 ; \eta 2=0.155)$. Follow-up analyses found that the MCI group produced slower latencies on serial position $1(F[1,31.67]=9.30, P<$ $0.005 ; \eta 2=0.170)$. A marginally significant effect suggesting slower latency on serial position $3(F[1,31.95]$ $=4.10 ; P<0.032 ; \eta 2=0.081$ ) was also found. Similar analyses for the 5 -span trial block were not statistically significant (Table 4).

Table 4. Symbolic working memory-latency for serial order recall

\begin{tabular}{llll}
\hline 4-Span (secs) & non- $\mathbf{M C l}(\boldsymbol{n}=\mathbf{3 3})$ & $\mathbf{M C l}(\boldsymbol{n}=\mathbf{2 4})$ & Significance \\
\hline position 1 & $2.47(0.93)$ & $3.71(1.82)$ & $P<0.001$ \\
position 2 & $1.30(1.31)$ & $1.81(0.94)$ & $\mathrm{ns}$ \\
position 3 & $1.26(0.80)$ & $1.95(1.55)$ & $P=0.032$ \\
position 4 & $0.64(0.27)$ & $0.75(0.28)$ & $\mathrm{ns}$ \\
\hline
\end{tabular}


Table 4. Symbolic working memory—latency for serial order recall (continued)

\begin{tabular}{llll}
\hline 5-Span (secs) & non-MCl & MCl & Significance \\
\hline position 1 & $2.60(1.25)$ & $2.57(1.48)$ & $\mathrm{ns}$ \\
position 2 & $1.30(1.05)$ & $1.49(0.99)$ & $\mathrm{ns}$ \\
position 3 & $1.70(1.14)$ & $2.21(2.03)$ & $\mathrm{ns}$ \\
position 4 & $1.66(0.94)$ & $2.34(1.22)$ & $\mathrm{ns}$ \\
position 5 & $1.03(1.26)$ & $1.33(1.30)$ & $\mathrm{ns}$ \\
\hline
\end{tabular}

ns: not significant

Intra-component latency and neuropsychological index scores

The regression analysis examining episodic memory test performance and 4-span intra-component latencies was significant $\left(\mathrm{R}=0.529, \mathrm{R}^{2}=0.280, F[4,51]=4.96, P<0.002\right.$; Table 5). The better episodic memory test performance was associated with faster latency on position 1 (beta $=-0.432, P<0.001$ ). A marginal relationship was found between better memory test performance and faster position 3 latency was also found (beta $=-0.256 ; P<0.049$ ). With regard to the naming/lexical access index, the regression analysis was significant $\left(\mathrm{R}=0.418, \mathrm{R}^{2}=0.175, F[4,52]=2.75, P<0.038\right)$. As with the episodic memory index, better neuropsychological test performance was associated with faster latency for position 1 (beta $=-0.339, P<0.017)$. Finally, the regression analysis examining executive functioning test performance and 4 -span intra-component latencies was also significant $\left(\mathrm{R}=0.529, \mathrm{R}^{2}=0.280, F[4,51]=4.95, P<0.002\right)$. Here, better executive test performance was associated with faster position 4 latency (beta $=-0.403, P<$ 0.002). None of the regression analyses for 5 -span intra-component latencies was significant.

Table 5. Regression coefficients: 4-span and 5-span serial order recall

\begin{tabular}{|c|c|c|c|c|c|}
\hline 4-span & $\begin{array}{l}1^{\text {st }} \text { response } \\
\text { backward }\end{array}$ & $\begin{array}{l}2^{\text {nd }} \text { response } \\
\text { backward }\end{array}$ & $\begin{array}{l}3^{\text {rd }} \text { response } \\
\text { backward }\end{array}$ & $\begin{array}{l}4^{\text {th }} \text { response } \\
\text { backward }\end{array}$ & \\
\hline \multirow[t]{2}{*}{ Executive index } & beta $=-0.253$ & beta $=-0.039$ & beta $=-0.031$ & beta $=-0.403$ & $\mathrm{n} / \mathrm{a}$ \\
\hline & $P<0.056$ & ns & ns & $P<0.002$ & \\
\hline \multirow[t]{2}{*}{ Language index } & beta $=-0.339$ & beta $=-0.138$ & beta $=-0.049$ & beta $=-0.008$ & $\mathrm{n} / \mathrm{a}$ \\
\hline & $P<0.017$ & ns & ns & ns & \\
\hline \multirow[t]{2}{*}{ Memory index } & beta $=-0.432$ & beta $=0.105$ & beta $=-0.256$ & beta $=-0.003$ & $\mathrm{n} / \mathrm{a}$ \\
\hline & $P<0.001$ & $\mathrm{~ns}$ & $P<0.049$ & $\mathrm{~ns}$ & \\
\hline \multirow[t]{2}{*}{ 5-span } & $1^{\text {st }}$ response & $2^{\text {nd }}$ response & $3^{\text {rd }}$ response & $4^{\text {th }}$ response & $5^{\text {th }}$ response \\
\hline & backward & backward & backward & backward & backward \\
\hline \multirow[t]{2}{*}{ Executive index } & beta $=0.068$ & beta $=-0.001$ & beta $=-0.208$ & beta $=-0.062$ & beta $=0.070$ \\
\hline & ns & ns & ns & ns & ns \\
\hline \multirow[t]{2}{*}{ Language index } & beta $=0.078$ & beta $=-0.008$ & beta $=-0.298$ & beta $=0.066$ & beta $=0.298$ \\
\hline & ns & ns & $P<\mathrm{ns}$ & ns & ns \\
\hline \multirow[t]{2}{*}{ Memory index } & beta $=-0.096$ & beta $=0.102$ & beta $=-0.267$ & beta $=-0.288$ & beta $=0.241$ \\
\hline & ns & ns & ns & ns & ns \\
\hline
\end{tabular}

ns: not significant; n/a: not available

\section{Discussion}

In prior research, Emrani and colleagues $[9,10]$ were able to operationally define the neurocognitive constructs underlying the theory of executive attention $[7,8]$ with analyses of latency data obtained on a digit span backward test. In the current research, we extended these findings and demonstrated that similar patterns of behavior can be captured using the Symbolic Working Memory subtest, a multi-modal digit reordering test where stimuli are initially administered verbally, but responses are captured via touch.

As reviewed above, working memory is a neurocognitive construct focused on the intention to act. The cognitive operations underlying working memory include collating all past experiences necessary to complete the task at hand. The construct of working memory "looks backward," recruiting long-term memoranda and prior experiences consistent with the task demands needed for goal directed activity. 
Fuster's [7, 8] construct of working memory is consistent with constructs suggested by Atkinson and Shiffrin [25], Baddeley [26], Norman [27] and Norman and Shallice [28]. Prior functional imaging data obtained from adults during a delay visual, non-spatial, match-to-sample task [29] found considerable activation involving bilateral dorsolateral prefrontal cortical regions during the initial trial epochs. In a series of studies [30] using a variety of verbal, auditory, and visual stimuli, bilateral dorsolateral prefrontal cortical regions along with posterior cortical regions consistent with the sensory modalities of the test stimuli tended to be highly active during initial trial epochs. However, during mid- and late-delay time epochs; and, immediately preceding the requested response; activation shifts to prefrontal and premotor regions, respectively [31; see ref 7, pp. 298-301, for a review]. These data have been interpreted to suggest that different, but highly integrated neurocognitive constructs predominant at different times during a participants response [7,8]. In this context, the theory of executive attention $[7,8]$ characterizes the entire time necessary to complete the task as a superordinate temporal gestalt. Subordinate to this temporal gestalt are three neurocognitive constructs - working memory, preparatory set, and inhibitory control. However, exact operations regarding how these three constructs operate are likely variable and contingent on the task under consideration.

On the Symbolic Working Memory Test, one must leverage memoranda to call into mind attributes associated with digits as well as the cognitive resources for mentally re-order digits as requested. Recalling or repeating a circumscribed string of digits or bits of information has become a generic, everyday experience (e.g., phone numbers, passwords, etc.). Therefore, the cognitive operations associated with generic instances involving serial order recall are likely recruited. Reasonably so, on correct 4-span test trials both groups (non$\mathrm{MCI}$ and $\mathrm{MCI}$ ) took their longest time to respond to the first number to be recalled. However, MCI patients required more time than non-MCI patients for position 1 suggesting that MCI patients needed to work harder to recruit the necessary resources to initiate a response.

Similar to the neurocognitive construct of working memory, one function of preparatory set is to focus on the intention to act. In addition to this role, preparatory set sustains the ability to respond consistently with task demands. Preparatory set is, therefore, attention "towards the future", i.e. attention focused on the prospective cognitive operations necessary to attain a goal. Evidence for this neurocognitive construct comes from electrophysiological research using delayed response tasks with primates. This research has documented the presence of slow, negative potentials (i.e. contingent negative variation; CNV) in anticipation to a motor act [32]. Further, electrophysiological activity has been shown to be proportional to the certainty that the respondent can predict a positive outcome [33]. In the current research, the latency in the middle of the test trial, i.e. position 3, was slower for MCI compared to the non-MCI patients.

Finally, inhibitory control is attention focused on excluding responses not germane to the task at hand. Any number of internal or external factors can derail correct serial order recall. Emrani and colleagues [9] noted that errors produced by MCI patients often occurred toward the latter portion of their response. Moreover, many of these errors, termed between-trial errors, were such that a digit from an immediately preceding test trial was incorrectly inserted into the current response. This type of error is consistent with recurrent perseverations as described by Sandson and Albert [34]. In addition to a slower latency in order to 'check in' with test demands, slower latency on position 3 may be suggestive of a concomitant, faulty inhibitory control processes, where MCI patients required more time to inhibit external information that would otherwise derail one from the task at hand. Past research suggests that the inhibition of information not germane to the task at hand is the province of the basal ganglia and orbital prefrontal cortical regions [33].

A parsimonious view of the latency data described by Emrani and colleagues $[9,10]$; and the data described in the current research, is to view the entirety of patients' response as an overall temporal gestalt whereby patients need to manage diverse test contingencies at different times during the generation of the response. This interpretation, while plausible, requires additional research. Data using imaging technology demonstrating the activation of different brain regions as a function of time-epoch in patients with suspected MCI would provide additional support for this model $[7,8]$. Finally, performance on the 
5-span trial was generally not statistically significant. The reason(s) for this are unclear but may revolve a restriction of range of data because the number of correct trials achieved.

Data supporting the notion that time-epochs when asked to mental order digits are related to integrated but differing underlying neurocognitive operations comes from the regression analyses described above. For example, faster position 1 latency was highly associated with better episodic memory and naming/lexical access test performance. An association between faster position 1 latency and better performance on episodic memory and naming/lexical access test comports with clinical observations. Indeed, a strategy used by many patients when asked to mentally re-order 4 or 5 bits of information is to subvocalize or actually rehearse the entire operation out loud, peeling numbers off numbers one at a time until the entire operation is completed. It is, therefore, reasonable to associate intact episodic memory and naming/lexical accessing skills with this portion of the test. On the other hand, faster position 4 latency, i.e. faster latency to bring the 4 -span test trial to fruition, was associated with better executive test performance. This observation is consistent that the notion that latter response latencies when asked to mentally reorder information may be related to both preparatory set or the ability to marshal the cognitive resources to sustain mental set; and, inhibitory control or the ability to inhibit the production of errors.

Clinical drug trials designed to treat AD have not been successful [35]. Greater attention is now devoted to designing pharmacological intervention for patients with $\mathrm{MCI}$ rather than dementia. Therefore, identifying patients as early as possible in an emergent dementia-related disease process is highly desirable. Coupling traditional neuropsychological tests with digital technology could yield a panel of neurocognitive biomarkers that are highly sensitive to emergent illness. Among any sample of potential participants for the treatment of prodromal dementia, it is possible that analyses of the time-based data could signal the presence of emergent illness even if the actual response was correct. More research is required to test this prediction.

The current research is not without weaknesses. Our sample size and some of the effects that were obtained was modest. Also, the structure of the Symbolic Working Memory Test with trial blocks containing only three test trials could have restricted the time-based data that was obtained. Future research using cross-modal test paradigms would benefit from tasks containing more test trials. However, the current research has several strengths including a validated method by which MCI and non-MCI groups were identified, and the novel use of comparatively inexpensive, simple, but powerful technology to obtained time-based data. In sum, the current research suggests that slower response latencies, in conjunction with correct serial order recall, can operationally define neurocognitive constructs underlying the theory of executive attention [7, 8]. Moreover, time-based parameters as described by Emrani and colleagues [9, 10], and data from the current research, could eventually constitute a set of neurocognitive biomarkers that may be used to tract emergent dementia and MCI syndromes.

\section{Abbreviations}

AD: Alzheimer's disease

MCI: mild cognitive impairment

SD: standard deviation

VaD: vascular dementia

\section{Declarations}

\section{Author contributions}

SE, ML, CCP, SB, VW, EM, RS, GB, \& DJL contributed to study concept and manuscript preparation; SE, VW, EM, DJL contributed to data acquisition; SE, ML, SB, VW, EM, GB, DJL contributed to statistical analyses; DJL, GB-funding recipients; All authors read and approved the submitted version. 


\section{Conflicts of interest}

The authors declare that they have no conflicts of interest.

\section{Ethical approval}

This study was conducted consistent with the Declaration of Helsinki.

\section{Consent to participate}

Not applicable.

\section{Consent to publication}

Not applicable.

\section{Availability of data and materials}

Not applicable.

\section{Funding}

The study was funded by New Jersey Health Foundation (ISFP 23-19); Osteopathic Heritage Foundation (2018002166). The funders had no role in study design, data collection and analysis, decision to publish, or preparation of the manuscript.

\section{Copyright}

(C) The Author(s) 2021.

\section{References}

1. Emrani S, Lamar M, Price CC, Wasserman V, Matusz E, Au R, et al. Alzheimer's/vascular spectrum dementia: classification in addition to diagnosis .J Alzheimers Dis. 2020;73:63-71.

2. Lamar M, Price CC, Giovannetti T, Swenson R, Libon DJ. The dysexecutive syndrome associated with ischaemic vascular disease and related subcortical neuropathology: a Boston process approach. Behav Neurol. 2010;22:53-62.

3. Libon DJ, Lamar M, Price CC, Jefferson AL, Swenson R, Au R. Neuropsychological evaluation for vascular dementia. In: Smith GE, Farias ST, editors. APA handbook of dementia. Washington: American Psychological Association; 2018. pp. 325-41.

4. Wong B, Lucente DE, MacLean J, Padmanabhan J, Quimby M, Brandt KD, et al. Diagnostic evaluation and monitoring of patients with posterior cortical atrophy. Neurodegener Dis Manag. 2019;9:217-39.

5. Kamath V, Sutherland ER, and Chaney GA. A meta-analysis of neuropsychological functioning in the logopenic variant of primary progressive aphasia: comparison with the semantic and non-fluent variants. J Int Neuropsychol Soc. 2020;26:322-30.

6. Lamar M, Price CC, Davis KL, Kaplan E, Libon DJ. Capacity to maintain mental set in dementia. Neuropsychologia. 2002;40:435-45.

7. Fuster JM. The prefrontal cortex. 4th ed. London: Academic Press; 2008.

8. Fuster, JM. Cortex and memory: emergence of a new paradigm. J Cogn Neurosci. 2009;21:2047-72.

9. Emrani S, Lamar M, Price CC, Wasserman V, Matusz E, Swenson R, et al. The thalamus and mental manipulation statistically-determined patients with mild cognitive impairment: a potential neurocognitive biomarker. Abstract presented at the $48^{\text {th }}$ annual meeting of the International Neuropsychological Society; 2020 Feb 5-8; Denver, USA. Salt Lake: International Neuropsychological Society; 2020.

10. Emrani S, Wasserman VJ, Baliga S, Matusz EF, Lamar M, Price C, et al. Working memory test performance in statistically-determined patients with mild cognitive impairment: an analysis of latency versus 
accuracy. Abstract presented at the $47^{\text {th }}$ annual meeting of the International Neuropsychological Society; 2019 Feb 20-23; New York, USA. Salt Lake: International Neuropsychological Society; 2019.

11. Lamar M, Price CC, Libon DJ, Penney DL, Kaplan E, Grossman M, et al. Alterations in working memory as a function of leukoaraiosis in dementia. Neuropsychologia. 2007;45:245-54.

12. Lamar M, Catani M, Price CC, Heilman KM, Libon DJ. The impact of region-specific leukoaraiosis on working memory deficits in dementia. Neuropsychologia. 2008;46:2597-601.

13. Jak AJ, Bondi MW, Delano-Wood L, Wierenga C, Corey-Bloom J, Salmon DP, et al. Quantification of five neuropsychological approaches to defining mild cognitive impairment. Am J Geriatr Psychiatry. 2009;17:368-75.

14. Sheslow D, Adams W. (2002). Wide range assessment of memory and learning. 2 nd ed. Administration and technical manual. Lutz (FL): Pearson. https://www.pearsonassessments.com/store/usassessments/ en/Store/Professional-Assessments/Cognition-\%26-Neuro/Wide-Range-Assessment-of-Memory-andLearning-\%7C-Second-Edition/p/100001702.html

15. Yesavage JA, Brink TL, Rose TL, Lum O, Huang V, Adey M, et al. Development and validation of a geriatric depression screening scale: a preliminary report. J Psychiatr Res. 1982;17:37-49.

16. Cummings JL, Mega M, Gray K, Rosenberg-Thompson S, Carusi DA, Gornbein J. The Neuropsychiatric Inventory: comprehensive assessment of psychopathology in dementia. Neurology. 1994;44:2308-14.

17. Edmonds EC, Ard MC, Edland SD, Galasko DR, Salmon DP, Bondi MW. Unmasking the benefits of donepezil via psychometrically precise identification of mild cognitive impairment: a secondary analysis of the ADCS vitamin E and donepezil in MCI study. Alzheimers Dement (N Y). 2017;4:11-8.

18. Emrani S, Libon DJ, Lamar M, Price CC, Jefferson AL, Gifford KA, et al. Assessing working memory in mild cognitive impairment with serial order recall. J Alzheimers Dis. 2018;61:917-28.

19. Bondi MW, Edmonds EC, Jak AJ, Clark LR, Delano-Wood L, McDonald CR, et al. Neuropsychological criteria for mild cognitive impairment improves diagnostic precision, biomarker associations, and progression rates. J Alzheimers Dis. 2014;42:275-89.

20. Thomas KR, Edmonds EC, Delano-Wood L, Bondi MW. Longitudinal trajectories of informant-reported daily functioning in empirically-defined subtypes of mild cognitive impairment. J Int Neuropsychol Soc. 2017;23:521-7.

21. Eppig J, Wambach D, Nieves C, Price CC, Lamar M, Delano-Wood L, et al. Dysexecutive functioning in mild cognitive impairment: derailment in temporal gradients. J Int Neuropsychol Soc. 2012;18:20-8.

22. Dixon WJ. Simplified estimation from censored normal samples. Ann Math Statist. 1960;31:385-91.

23. Wilkinson GS, Robertson GJ. (2006). Wide Range Achievement Test. 4th ed. Lutz (FL): Pearson. https:// www.pearsonassessments.com/store/usassessments/en/Store/Professional-Assessments/AcademicLearning/Wide-Range-Achievement-Test-\%7C-Fourth-Edition/p/100001722.html

24. Folstein MF, Folstein SE, McHugh PR. "Mini mental state": a practical method for grading the cognitive state of patients for the clinician. J Psychiatr Res. 1975;12:189-98.

25. Atkinson RC, Shiffrin RM. Human memory: a proposed system and its control processes. In: KW Spence and JT Spence, editors. The psychology of learning and motivation: advances in research and theory. Vol. 2. New York: Academic Press; 1968. pp. 89-195.

26. Baddeley AD. Working Memory. Oxford: Clarendon Press; 1986.

27. Norman DA. Toward a theory of memory and attention. Psychol Rev. 1968;75:522-36.

28. Norman DA, Shallice T. Attention to action. In: Davidson RJ, Schwartz GE, Shapiro DE, editors. Consciousness and self-regulation. New York: Plenum; 1986. pp. 1-18.

29. Swartz BE, Halgren E, Fuster JM, Simpkins TF, Gee M, Mandelkern M. Cortical metabolic activation in humans during a visual memory task. Cerebral Cortex. 1995;5:205-14. 
30. Buchsbaum BR, Olsen RK, Koch P, Berman KF. Human dorsal and ventral auditory streams subserve rehearsal-based and echoic processes during verbal working memory. Neuron. 2005;48:687-97.

31. Libert B, Wright EW Jr, Gleason CA. Preparation- or intension-to-act, in relation to pre-event potentials recorded at the vertex. Electroencephalogr Clin Neurophysiol. 1983;56:367-72.

32. Abplanalp JM, Mirsky AF. Electroencephalographic correlates of delayed-alternation and visual discrimination learning in rhesus monkeys. J Comp Physiol Psychol. 1973;85:123-31.

33. Aron AR, Poldrack RA. The cognitive neuroscience of response inhibition: relevance for genetic research in attention-deficit/hyperactivity disorder. Biol Psychiatry. 2005;57:1285-92.

34. Sandson J, Albert ML. Varieties of perseveration. Neuropsychologia. 1984;22:715-32.

35. Cummings JL, Morstorf T, Zhong K. Alzheimer's disease drug-development pipeline: few candidates, frequent failures. Alzheimers Res Ther. 2014;6:37.

36. Heaton RK, Miller SW, Taylor MJ, Grant I. (2004). Revised comprehensive norms for an expanded Halstead-Reitan Battery: demographically adjusted neuropsychological norms for African American and Caucasian adults. Lutz (FL): Psychological Assessment Resources. https://www.parinc.com/products/ pkey/357

37. Wechsler D. WAIS-III: administration and scoring manual: Wechsler adult intelligence scale. 3rd ed. San Antonio (TX): Psychological Corporation; 1997.

38. Delis DC, Kramer JH, Kaplan E, Ober BA. (2000). California Verbal Learning Test. 2nd ed. Pearson. https:// www.pearsonassessments.com/store/usassessments/en/Store/Professional-Assessments/Cognition\%26-Neuro/California-Verbal-Learning-Test-\%7C-Second-Edition/p/100000166.html 